\title{
ANALYZING CLOSER CARE STRATEGIES FOR ELDERLY PATIENTS: EXPERIENCE AND REFLECTIONS FROM MODELING WITH SYSTEM DYNAMICS
}

\author{
Dr. Gary Linnéusson \\ School of Engineering \\ Jönköping University \\ P.O.Box 1026, SE-551 11, Jönköping, Sweden \\ gary.linneusson@ju.se
}

Dr. Ainhoa Goienetxea Uriarte

School of Engineering Science

University of Skövde

P.O.Box 408, SE-541 28, Skövde, Sweden

ainhoa.goienetxea@his.se

\begin{abstract}
The expected demographic changes, and especially the rise in life expectancy, will considerably increase elderly patients' demand for healthcare. There are different strategies that can offer better care for these patients, reduce their unnecessary visits to the emergency departments, and in consequence, reduce the number of hospitalizations and days at the hospital. This study employed system dynamics to analyze the economic and quality-related effects of different closer care strategies such as investments in care coordinators and mobile health clinics, as well as to offer proactive care in the primary care facilities for elderly patients. The results indicate that a combination of the different strategies will support better care for patients, will reduce hospital costs and will reduce the existing pressure on the emergency department. The paper also reflects on the process followed to conduct the study and the lessons learned.
\end{abstract}

Keywords: System Dynamics, Elderly Care, Simulation, Healthcare

\section{INTRODUCTION}

There is an increasing pressure on healthcare policymakers to design systems that will be sustainable in the future (Lyons and Duggan, 2015). This is a challenging task, taking into account the expected increase of the aging population, e.g., the actual world population aged sixty years or over will be doubled by 2050 (United Nations, 2017). Additionally, the rates of chronical diseases and multimorbidity are expected to increase in elderly patients (Lindgren, 2016), which in consequence will increase the demand on the healthcare system and the economic pressure on healthcare providers.

According to Lyons and Duggan (2015), there are different factors that characterize the healthcare infrastructure: 1) exogenous factors associated with population dynamics (demographics, lifestyle, etc.); and 2) internal decision variables associated with policy measurements as well as the development of the healthcare services to respond to the existing demand by the exogenous factors. This paper focuses on the analysis of the second factor and tries to analyze the impact of establishing policies and to develop the healthcare services to offer a closer and better care for elderly patients ( 65 years or older), and at the same time, to minimize the care they require from the emergency departments (ED) and the subsequent hospitalizations and days staying at the hospital. Different authors have defended this approach stating that to offer timely and effective primary care (PC) can even reduce hospitalizations, and thereby, avoidable complications during hospitalizations for these type of patients (Boyd et al 2008).

There are different operational research methods and tools that can be employed to support healthcare policymakers to make better decisions, some of them are reviewed in Hulshof et al. (2012). Simulation is a popular technique, and different studies employing simulation to support healthcare system design and improvement have been reviewed by different authors (Brailsford et al 2009; Katsaliaki and Mustafee, 2011; Mielczarek and Uziałko-Mydlikowska, 2012; Salleh et al 2017). When the problem under study has a dynamic nature and there is a need to understand the 
interconnections between the different parts involved in the system, System Dynamics (SD) is an appropriate tool to be employed (Linnéusson et al 2018). As discussed by Senge and Sterman (1992), it enables multiple testing with the objective to question own mental models, and at the same time, it questions the underlying values governing the system. Different authors have studied the dynamic complexity of restructuring the healthcare systems via SD, such as in Homer and Hirsch (2006), as well as in the examples provided in the reviews by Kunc et al (2018) and Chang et al (2017).

This paper presents the results of a case study using SD with the aim of analyzing the effects of the development of closer care strategies for elderly patients including multimorbidity patients and frequent attenders (FA). To the best of the authors' knowledge, this approach has still not been used to analyze the dynamics of this problem. Besides the traditional case study report, this paper also presents a reflection which analyses the process and learnings from the multiple trial and error search for a problem focus, subsequent problem behavior to be modeled, its consequences to the model building process, and how the results supported decision-making.

The article is structured as follows: Section 2 presents the background on how and why the project was done; Section 3 describes the method and steps applied to conduct the project; Section 4 elaborates on the details about the qualitative SD model; Section 5 briefly presents the quantitative SD model and overall simulation results; Section 6 includes a reflection and lessons learned during the project development; finally, Section 7 reveals the conclusions and future work.

\section{BACKGROUND}

As an important step towards quality in care, the region of Västra Götaland (VGR) in Sweden is working on an initiative to offer closer care to patients. This includes four areas of action: 1) to develop the organization to offer closer care; 2) to concentrate the offered care to achieve better quality and availability; 3) to develop digital care services; and 4) to focus on quality-driven improvements. Some of the main motivations to work with closer care actions are related to increase the quality of care provided, as well as to decrease existing waiting times, queues, and rising costs for hospitals (Taylor and Dangerfield, 2005). The lack of coordination and availability, as well as a reactive and non-person-centered focus which usually has characterized PC, has influenced the behavior of patients that prefer to go to the ED, sometimes unnecessarily.

Elderly patients are a specific group of patients who have continuous care need and contribute with a considerable amount of visits to the ED and hospitalizations (LaCalle and Rabin, 2010). According to data from 2016, elderly people (65 or older) in VGR were around 320.000 . Of these, around $14 \%$ were patients with multimorbidity, and around $1,56 \%$ of them were FA in the ED, which means that they visited the ED at least four times in one year (the most common definition of FA, according to LaCalle and Rabin, 2010). The details of these patients are shown in Table 1.

Table 1 Data about elderly patients' non-planned hospital services usage.

\begin{tabular}{|c|c|c|c|}
\hline Parameter & $\begin{array}{c}\text { Multimorbidity frequent } \\
\text { attender patients }\end{array}$ & $\begin{array}{l}\text { Multimorbidity patients } \\
\text { - non frequent attenders }\end{array}$ & $\begin{array}{c}\text { Non-multimorbidity } \\
\text { patients }\end{array}$ \\
\hline Total persons in the region & 5.000 & 41.000 & 274.000 \\
\hline Average amount of ED visits per year & 5,5 & 0,9 & 0,3 \\
\hline $\begin{array}{l}\text { Average amount of times the patient is hospitalized } \\
\text { per year }\end{array}$ & 3,3 & 0,7 & 0,1 \\
\hline Average amount of days per hospitalization & 6 & 10 & 6 \\
\hline$\%$ of patients with avoidable hospitalization & \multicolumn{2}{|c|}{$15 \%$} & $2 \%$ \\
\hline $\begin{array}{l}\% \text { of patients returning to the ED and being } \\
\text { hospitalized again after } 1-30 \text { days }\end{array}$ & \multicolumn{2}{|c|}{$5 \%$} & $1 \%$ \\
\hline
\end{tabular}

Even if the elderly are a relatively small group of patients, the number of visits to ED, hospitalization rates and length of stay at the hospital are considerably high in comparison to other groups. These variables have been identified as one of the major causes of ED overcrowding (Moskop et al 2009). Therefore, analyzing how to offer closer care for this group of patients was prioritized.

Multimorbidity FA patients within this group have the highest number of visits to the ED, hospitalization rates, as well as the highest avoidable hospitalization rates. The data show that many of these patients were hospitalized unnecessarily and that a considerable percentage of them were coming back to the ED in the period of a month. 
Different actions to offer closer care performed in specific municipalities of the region reported very good results in terms of quality of care provided and cost savings. For example, to have care coordinators in charge of coordinating the care offered to elderly patients arriving to the ED together with the community-based services and PC, as well as to introduce a survey about the status of the elderly patients, helped reduce the amount of time the patients were waiting in the ED, reduced the number of patients being hospitalized, and reduced even the number of patients returning back to the ED within a month. Another tested action, which proved to reduce the number of visits to the ED, is to have mobile health clinics including a team of a doctor and nurses who visit elderly patients in their homes or home care facilities. These patients are usually very unstable or need palliative care. The mobile health clinics try to offer proactively high-continuity and person-centered care, as opposed to the commonly reactive care offered both in the ED and PC. A third action that proved to reduce the number of visits to the ED is to proactively work with the elderly in the PC facilities. Meaning that PC visits are pre-booked systematically and more time is assigned to doctors and nurses to meet and treat these patients. In a specific region of Sweden, this proved to reduce $20 \%$ of the visits of this group of patients to the ED reducing, in consequence, the number of hospitalizations.

Therefore, the objectives pursued in this project were: 1) to analyze the dynamics of elderly patients' care-seeking behavior; 2) to analyze how different actions to offer closer care can impact the number of visits in the ED and hospitalizations; and 3) to analyze the effect in the costs.

\section{WHICH STEPS DID WE TAKE?}

The steps followed to conduct the project were the ones presented in Figure 1.

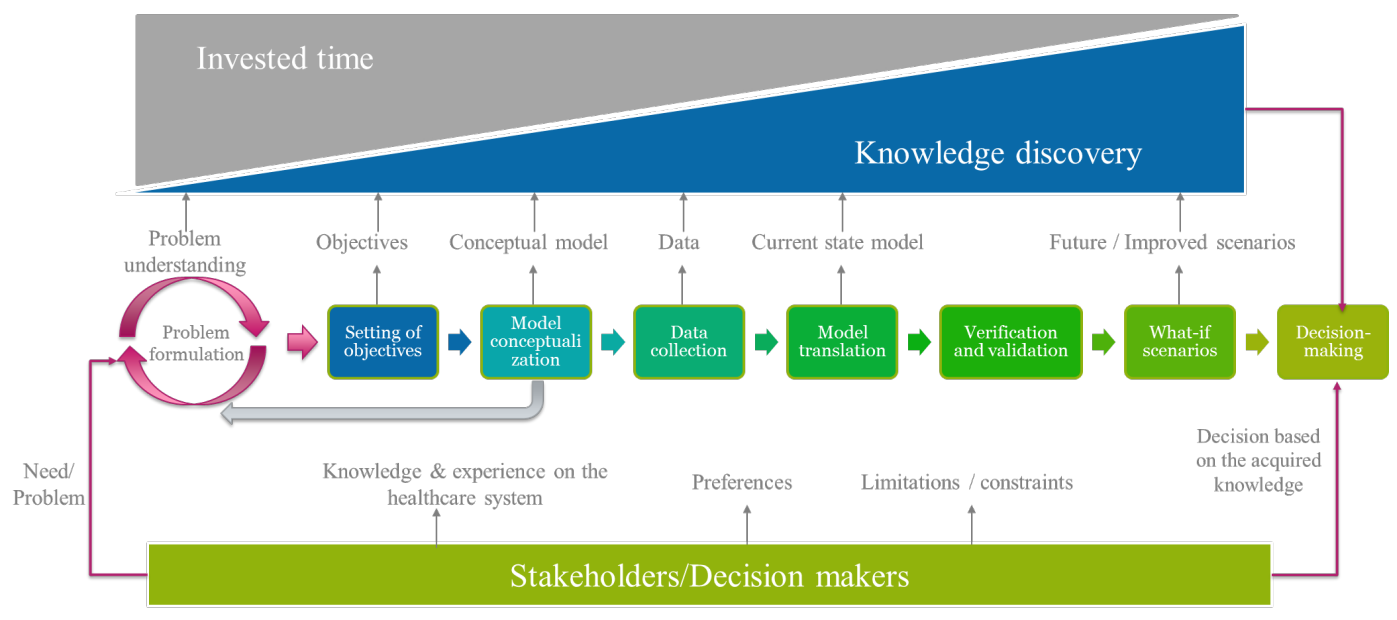

Figure 1 The process followed to conduct the study. Updated from Goienetxea Uriarte et al (2017).

The project started with an open problem formulation about how to define closer care for all the patients in the region. To better understand this concept a workshop was organized with the stakeholders and subject matter experts from the PC, ED, and the hospital. Additionally, many iterations were performed in group model building sessions to facilitate discussions about what does it mean to offer closer care, who needs it, what is the dynamic behavior of patients seeking care, etc. These discussions eventually helped to focus the study on elderly patients visiting the ED. After deciding the problem to be addressed, the modelers together with the stakeholders developed the conceptual model using causal loop diagramming (CLD) described in section 4. In the next step, a more concrete focus was decided where specific closer care strategies for elderly patients were chosen to be analyzed via SD, these included: 1) to have care coordinators in all the ED of the region; 2) to have enough mobile care clinics to be able to offer the service to all in need; and 3) to offer a proactive care in the PC facilities. Subsequently, data was gathered and the current state simulation model was developed, as described in section 5. The model was verified and validated together with the stakeholders, based mainly on historical data and face validation (Sargent, 2011). Then different scenarios related to the three closer care strategies explained previously were developed, compared, 
and combined to analyze their impact in reducing the visits to the ED, number of hospitalizations and days at the hospital, as well as costs. The decision makers were then provided with the results which served as an input to decision-making regarding how to conduct the investments for better closer care in the region.

The initial stages of the process, especially the ones related to problem formulation, the setting of objectives and model conceptualization took a considerable amount of time from the project budget, although it was on the last stages of the project where most of the knowledge was generated.

\section{ANALYZING THE DYNAMICS OF ELDERLY PATIENTS IN THE HEALTHCARE SYSTEM USING CAUSAL LOOP DIAGRAMMING}

The closer care concept was considered a general fix to the healthcare system and to support reducing the care-seeking behavior at EDs in specific. However, in order to comprehend how the pressure on EDs could be released, a CLD was developed with the purpose to identify the potential feedback mechanisms involved in creating this pressure. The problem structuring process explored several potential problems eventually resulting in the CLD presented in Figure 2, which established a creative dialogue and a deeper understanding of the problem dynamics. It was the initial articulation of the central feedback loop of R1 which finally released the dialogue. The model includes several identified short- and long-term feedback of events and actions that together create the dynamic forces of central importance to better understand the complexity and inherent challenges of improving the quality of care and its related costs. The core of the CLD is composed of two central feedback loops:

- $\mathrm{R} 1$ - GoTo ED 65+: the main feedback resulting in elderly patients' care-seeking behavior, which creates a sustaining pressure on EDs.

- B1 - Proactive Care 65+: the main feedback which could potentially limit or enable the development of elderlies' current care-seeking behavior to the ED.

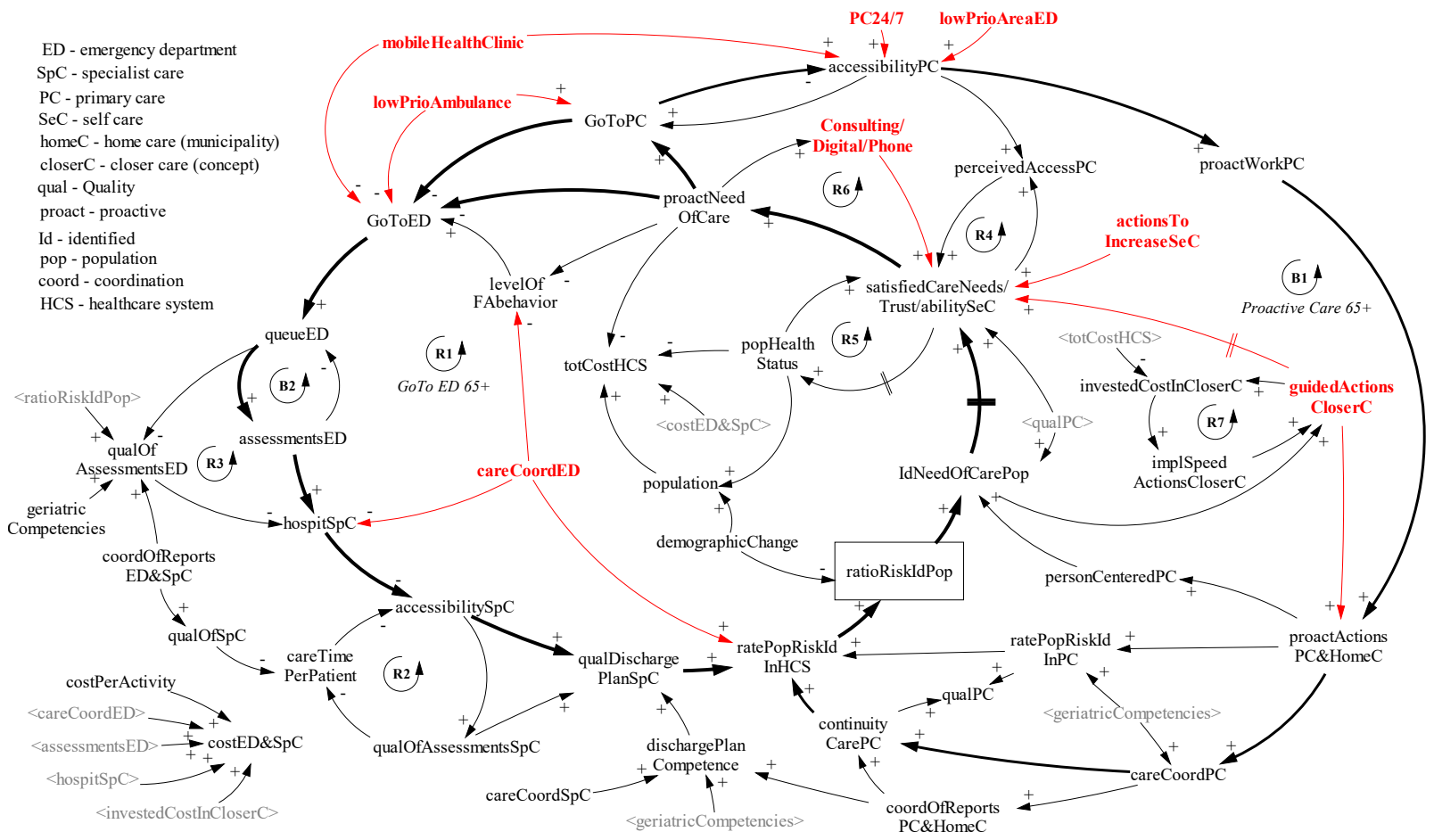

Figure 2 The main interactions between the healthcare system and elderlies use of care, using Vensim software.

The variables employed in the CLD are represented by abbreviations described in the legend in Figure 2. In the description below, a subset of these variables and their feedback loops are elaborated using Italics.

The R1-loop considers how to reduce the current care-seeking behavior to the ED (GoToED). R1 is a reinforcing feedback loop that can potentially lead to a vicious cycle towards a worse-and-worse 
condition, or a virtuous cycle towards a better-and-better condition. Hence, if GoToED could be reduced, a chain-reaction of positive effects could be expected such as 1) queueED would be reduced, 2) lesser assessments at the ED (assessmentsED) would be needed, and 3) fewer people would need hospitalization and specialist care (hospitSpC), which would increase the accessibility of hospitals (accessibility $S p C$ ) and potentially improve the quality of the discharge planning (qualDischargePlanSpC) leading to better care. However, the opposite is also considered to hold true, i.e., lower accessibility affects time invested in discharge planning leading to a cutting-the-cornerbehavior (Repenning and Sterman, 2001).

Another key aspect brought up at the very first workshop is that in order to provide more efficient and quality care there is a dependency on the current levels of the patients' trust in the healthcare system (R1-loop). In succeeding discussions it was considered that trust was closely connected to satisfied patient care needs, to increase their health literacy, as well as their self-care. Hence, the variable with the name satisfiedCareNeeds/Trust/ability SeC, is a multidimensional variable with the outcome of changing the conditions for proactiveness in the healthcare system. It is also a variable strengthened and weakened by several other feedback loops, where the desired improved outcome is to redirect people from the ED to more proactive ways to seek care. One fundamental aspect that supports raising the levels of satisfiedCareNeeds/Trust/abilitySeC is a parameter called risk identification. It is a term that represents when a person becomes a registered patient in the healthcare system. Meaning, that a person could have repeated interaction with the healthcare system without being risk identified, which could be the cause for becoming an FA (Kivelä et al 2018). This could be caused by the system itself through lacking quality in ED, PC, and the hospitals' discharge planning described above. Hence, actions to increase the rates of risk identifying patients in the healthcare system (ratePopRiskIdInHCS) are crucial to increase the number of people being identified (ratioRiskIdPop) and to reach its tipping point towards a more proactive state (wherever that might be). Moreover, ratioRiskIdPop is a level diluted by the continuous rates of demographic change, through deaths and new elderly people. Hence, the stock of risk identified people in the system and its connected flows are also important in order to attain the satisfied patient care needs for the population overall (IdNeedOfCarePop).

Another part of the healthcare system with the mission to provide proactive care found in the B1loop is the work carried out by the PC as well as by the municipalities through home care services. However, the level of proactive work (proactWorkPC) depends on the applied resources and resulting accessibility to PC (accessibilityPC), leading to more proactive actions in PC and home care (proactActionsPC\&HomeC). Proactive care includes three main actions: 1) higher levels of coordinated work in PC (careCoordPC) leading not only to better reporting between PC and home care (coordOfReportsPC\&HomeC) and supporting discharge planning at the hospitals, but also to improved continuity care in PC (continuityCarePC) which leads to higher precision in the risk identification of patient needs and improved quality of PC; 2) higher rates of patients being risk identified by the PC (ratePopRiskIdInPC), increasing the rate of overall risk identification in the healthcare system leading to improved quality where a higher quality of PC (qualPC) supports improving IdNeedOfCarePop and directly supports the level of satisfiedCareNeeds/Trust/abilitySeC; and 3) improved person-centered care (personCenteredPC), which directly supports higher quality care for those who are risk identified through more or less continuous monitoring.

Hence, the B1-loop identifies that proactiveness in PC is dependent on that resources are available and utilized according to the above-mentioned actions. Here, as an example, a changed balance of people who are seeking care, from GoToED to GoToPC, due to a productive implementation of higher quality actions in ED and hospitals can be restricted by a limiting performance of the B1-loop. Accordingly, improvements are needed in both the R1- and B1-loops to achieve sustainable effects on peoples' care-seeking behavior (proactNeedOfCare).

$\mathrm{R} 1$ and B1 are not the only loops in the diagram. Worth mentioning is the R5-loop, including variables decoupled through a time delay, where the variable popHealthStatus can be identified as a slow-working buffer affecting the overall performance of the healthcare system. In a well-functioning healthcare system (high levels of quality and proactive work as well as high levels of popHealthStatus) short-term cost savings may for a period cause economic benefits. Meanwhile, the consequences of mistreatment in the healthcare system could be hidden for the decision makers - and 
the system performance! - which on the longer term will likely reach a tipping point and the general popHealthStatus will be pushed to its threshold value where an escalating vicious cycle of a worseand-worse behavior in the R1-loop could be activated. While, in an already dysfunctional system, the power of inertia from the R5-loop may diminish the results from actions to improve the R1-loop. Hence, the popHealthStatus is potentially the memory of the healthcare system performance, which creates inertia between actions and their effects toward the desired proactive development.

Finally, different actions to offer closer care are represented by the red variables in the CLD, where some were recently implemented in part of the studied region as described in the background, and others are still in their design process. Identifying their interaction with the current system dynamics in the CLD visualizes where these actions may provide support to the desired proactive behavior. At some local hospitals, to have care coordinators in the ED (careCoordED) has provento reduce unnecessary hospitalizations, support the rate of risk identifications, and to identify pre-risk patients with potential escalating FA behavior. MobileHealthClinics also reactively absorb some of the care users which would otherwise GoToED, and provide closer care as a substitute to GoToPC having the effect of improving accessibility $P C$. At the same time, lowPrioAmbulances go to patients' home offering basic emergency care which reduce people GoToED and send patients on follow-upcontrols in PC increasing people who GoToPC. Moreover, to offer closer care could include using more local walk-in centers to increase the accessibility of service for low acuity emergency care, as well as having PC open 24/7 in order to improve the accessibility $P C$. Both are actions to improve the perceivedAccess $P C$ and relieve the pressure on the daily work of PC. These support maintaining the required levels of practWorkPC. Similarly, the actionsToIncreaseSeC would seemingly have a direct effect on increasing ability $S e C$, yet, having in mind its complex interaction with, e.g., the popHealthStatus which potentially can limit peoples' receptiveness, and thus, the success of such strategy.

Besides several more potential consequences, when analyzing the qualitative CLD using mental simulation, we can conclude that pulling the healthcare system into a more proactive balance through the aforementioned improvements will lead to a redistribution of how the total costs sum up (totCostHCS); which is a function of how care users seek care $(\operatorname{costED\& SpC})$, strongly affected by the current level of proactNeedOfCare, the amount of care users (population), and the popHealthStatus which altogether affect care users to live longer healthier lives.

\section{CALCULATING THE EFFECTS OF THE DIFFERENT CLOSER CARE STRATEGIES WITH SD}

The CLD provided with a systemic common view of the dynamics of elderly care users in the healthcare system and supported processing potential focuses of the SD model. The project group considered that all aspects could not be included in a simulation model due to insufficient data and time. One critical group of care users were elderly with multimorbidity and an FA behavior. To include the appropriate dynamics of this group, all the elderly population in the region was considered as part of the model population. Initially, the focus was on identifying the dynamics of how the elderly got into an FA behavior, but data was hard to identify and the project team experts considered current knowledge and research to be insufficient to its support. Instead, a stock and flow structure was developed of the elderly and their multimorbidity behavior, creating a dynamic theory that challenged the limited one-year perspective that mere statistical data provided. Moreover, three target groups could be identified from studying the data: Gr1) elderly without multimorbidity; Gr2) elderly with multimorbidity; and Gr3) elderly with multimorbidity having an FA behavior which is a subset of near $11 \%$ of all the elderly with multimorbidity. The elderly without multimorbidity with an FA behavior were so few they could be completely omitted as a group in the model.

The base structure (BS) of the model includes three parts as shown in Figure 3. These are: BS1) care demand based on elderlies multimorbidity behavior; BS2) calculations of the number of assessments at the ED, subsequent hospitalizations, and days at the hospital for the three defined target groups; and BS3) the estimated costs from the care usage. The model includes three additional parts to incorporate the scenarios of the identified closer care improvement actions: S1) to introduce care coordinators in the ED which identify elderly risk patients coming to the ED; S2) to promote the use of mobile health clinics which reduce unnecessary visits to ED and also hospitalizations; and S3) 


\section{Linnéusson and Goienetxea Uriarte}

to offer a proactive PC service in terms of more thorough consultations to elderly patients which was expected to lead to reductions in the visits to the ED.

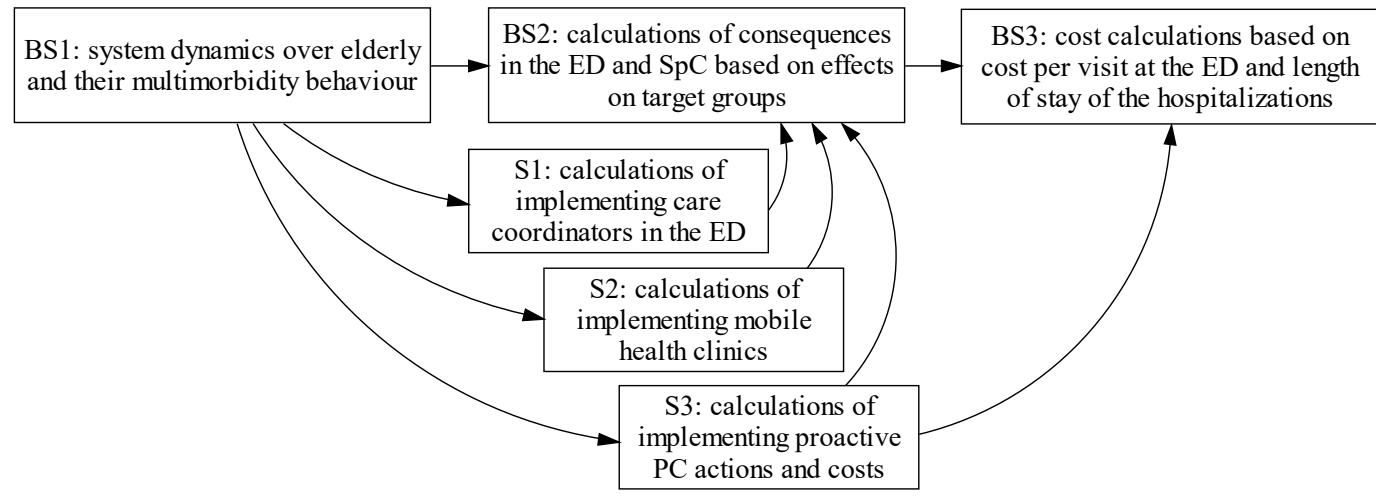

Figure 3 Simplified overview of the parts in the SD model, using Vensim software.

Additional information is found in table 2, in which the target groups included in the different scenarios are presented, as well as the results on the employed approximate consequences to the number of visits to the ED, the subsequent hospitalizations, and effects on the length of stay. Regarding costs to implement the tested scenarios, S1 and S2 were already funded by the region in previous years, thus no further investments were needed, while S3 included trading the added resources and costs in PC to the calculated cost benefits.

Table 2 Overview of data and results of the tested scenarios.

\begin{tabular}{|c|c|c|c|c|c|}
\hline & Target group (TG) & Visits to the ED & Hospitalizations & Length of stay & Increased cost \\
\hline S1 & $\begin{array}{c}11 \% \text { of } \\
(0.1 * \mathrm{Gr} 1+\mathrm{Gr} 2+\mathrm{Gr} 3)\end{array}$ & $\begin{array}{l}\text { no direct effect, } \\
\text { reduction of revisits }\end{array}$ & $\begin{array}{l}\sim 30 \% \text { reduction of which } \\
83 \% \text { had no hospitalization }\end{array}$ & same & already funded \\
\hline S2 & $\mathrm{Gr} 2$ & $\sim 0.9 \rightarrow 0.8 /$ person\&year & \multirow{3}{*}{$\begin{array}{l}\text { follows reductions } \\
\text { of the ED visits }\end{array}$} & $\sim 7 \%$ increase & already funded \\
\hline $\mathrm{S} 2$ & Gr3 & $\sim 40 \%$ reduction & & same & already funded \\
\hline S3 & $0.1 * \mathrm{Gr} 1+\mathrm{Gr} 2+\mathrm{Gr} 3$ & $20 \%$ reduction & & same & $2 *$ PC visits for $\mathrm{TG}$ \\
\hline
\end{tabular}

Figure 4 displays the simulation results in two inclusive parameters, to the left totaling the costs of the healthcare system and to the right the sum of all the visits to the EDs. An implementation period of three years was utilized. The graphs present the current scenario (line 4) together with the tested scenarios S1 (line 1), S2 (line 2) and S3 (line 3), and the combination of the scenarios (line 5). With respect to costs (to the left), the combination of scenarios S5 is the most beneficial, followed by $\mathrm{S} 3$. With respect to quality in care (to the right) leading to lower demand on the EDs, the effect of S2 and $\mathrm{S} 3$ is similar, while the combination of scenarios in line 5 is again the most beneficial.
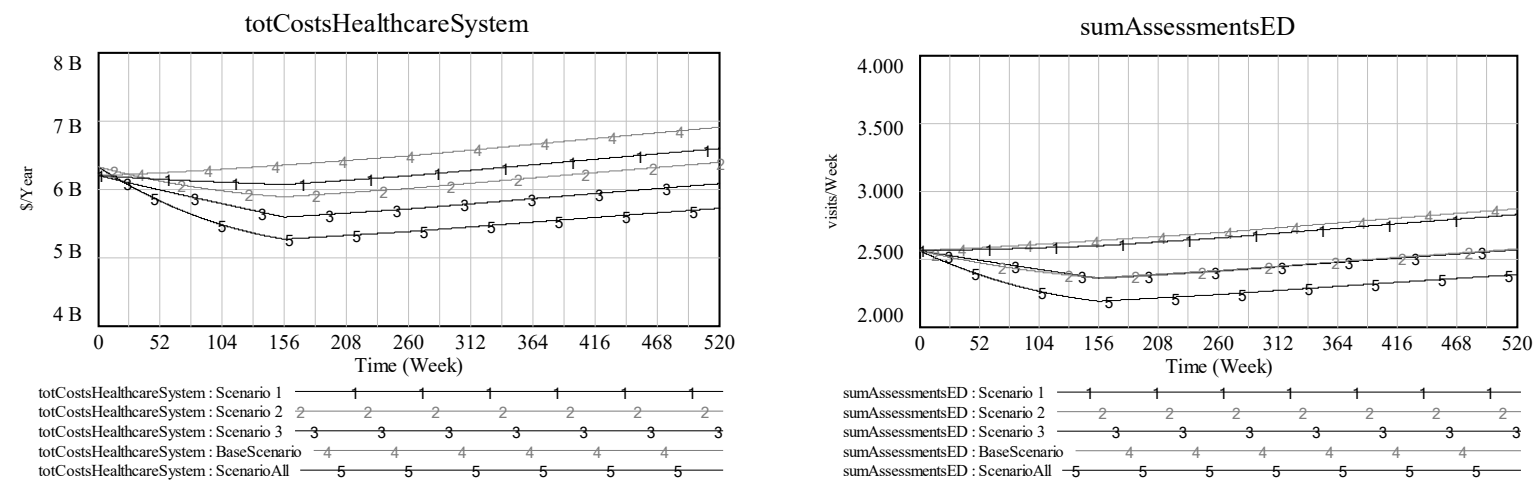

Figure 4 Result graphs of the tested scenarios on the total cost outcome and care-seeking behavior. 


\section{REFLECTING UPON THE PROCESS AND RESULTS. WHAT DID WE LEARN?}

This section describes some reflections about the problems encountered during the development of the simulation model, as well as some lessons learned that may serve as tips to those working with SD in similar projects in the healthcare domain. These are:

- Working with stakeholders with no experience with simulation: This was the first time the stakeholders worked in an SD simulation study (although they had previous experience with discrete event simulation - DES). This impacted mainly on the problem definition and structuring phase increasing the difficulty to focus the problem to be studied. This was due to the complexity of the problem, but also to the knowledge gap on defining dynamic feedback problems using SD. It was also evident that the expectations about the capabilities of simulation to support decisionmaking were unrealistic, as a decision maker pointed out: "I hope that with this study you can solve all the problems we have at the ED". Probably a good idea before being involved in a simulation study with non-experienced stakeholders is to provide education to decision makers on the capabilities of the method.

- Is SD too abstract to be understood?: SD was chosen for its capabilities of including feedback, studying short- and long-term dynamics, and due to the system-wide perspective of the problem at hand. However, when building the CLD and later on the quantitative model, it was evident that working with SD requires abstraction capabilities from the stakeholders that are not needed with, e.g., DES. This is probably something that should be explained and presented to stakeholders before starting an SD study.

- Qualitative vs. quantitative: The systemic nature of SD which allows the inclusion of qualitative and quantitative parameters makes it a very complete and flexible method, however, the experience from this project demonstrated that the stakeholders were not comfortable of including qualitative parameters that lacked proved evidence or statistical data to support the assumptions. This greatly limited the completeness of the model.

- Time invested vs. knowledge gained: As Figure 1 shows, the biggest gain in knowledge acquisition comes in the latter stages of the model development process. Especially when different what-if scenarios can be tested to see their potential benefits and drawbacks. However, due to the extensive amount of time taken to define the problem to be studied, as well as continuous changes in the focus of the scenarios to be analyzed, very little time was left to build complete what-if scenarios and make a deeper analysis of the results obtained. Therefore, how the model was used was very limited in comparison to the potential the model could have had to support decision-making.

- Trying to understand the problem vs. wanting a specific solution: An additional benefit of using $\mathrm{SD}$ is to provide a base for rich discussions including a system perspective. This provides a deep understanding of the problem under study and variables involved, which may be a good base for decision-making. However, the experience in this project showed that the decision makers' priority was not on understanding the problem but on getting a specific solution to the problem instead. In consequence, the CLD was very useful for the project team to understand the problem and the dynamics of the demand for care of the elderly, however, it was not employed by decision makers in their decision-making process. Instead, the decision makers just pursued specific results regarding a reduction in the number of visits to the ED and the number of hospitalizations, as well as the economic gain and loss depending on the scenario tested. This could have been done probably with advanced calculation worksheets instead of using an SD model.

- Healthcare domain specifics: There are still many barriers to overcome for extended use of simulation in the healthcare context. More experiences like the one presented in this paper are surely needed as an addition to courses or training to healthcare personnel, decision makers, and policy makers to show the potential of the method to support decision-making.

\section{CONCLUSIONS}

This paper describes a case study where SD has been employed as a method to model and analyze the demand for care of elderly patients. The objectives pursued with the study included an evaluation of different scenarios to offer closer care to these patients in order to increase the quality of care 
provided, to minimize the number of visits to the EDs, as well as to minimize the subsequent hospitalizations. An additional objective involved the analysis of the cost of these scenarios. In order to achieve these objectives, a qualitative model was designed which was valuable to open up discussions and to define and limit the scope of the project. Additionally, a quantitative model was also developed to test the economic effect of applying these closer care scenarios, which were 1) the implementation of care coordinators in the ED; 2) the implementation of mobile health clinics; and 3) employing proactive care in PC. It showed that the best results were provided by combining all three scenarios into a forth scenario. However, this combined scenario had less total benefit than adding the separate results from the scenario 1 to 3 due to the overlap effects from the closer care actions on the identified target groups of the elderly applied in the model.

In addition to the description of the process and results of the case study, this article also reflects on the journey of developing the SD model as well as the lessons learned, which may serve as an input to other simulation modelers working in similar projects in the healthcare domain or with stakeholders without experience with simulation projects.

The project results are being analyzed and employed as a support for decision-making on where to invest to offer closer and better care for elderly patients, which is even economically sustainable.

Further collaboration to analyze other existing problems and improvement areas in the regional healthcare system are nowadays under analysis, and simulation will surely be one of the methods considered to be employed to support the decision makers in these tasks.

\section{ACKNOWLEDGMENTS}

The authors gratefully acknowledge the region of Västra Götaland (VGR) for funding the project. The authors are especially thankful to the project group members from the Data analysis department of VGR Catarina Karlberg, Anna Norman Kjellström, Karin Fröjd, and Malin Lönnbark for their engagement during the whole development of the project.

\section{REFERENCES}

Boyd C M, Ritchie C S, Tipton E F, Studenski S A and Wieland D (2008). From Bedside to Bench: summary from the American Geriatrics Society/National Institute on Aging Research Conference on Comorbidity and Multiple Morbidity in Older Adults. Aging Clin Exp Res 20(3):181-8.

Brailsford S C, Harper P R, Patel B and Pitt M (2009). An analysis of the academic literature on simulation and modelling in health care. Journal of Simulation 3(3):130-140. doi: 10.1057/jos200910.

Chang A Y, Ogbuoji O, Atun R and Verguet S (2017). Dynamic modeling approaches to characterize the functioning of health systems: A systematic review of the literature. Social Science and Medicine 194:160-167. doi: 10.1016/j.socscimed.2017.09.005.

Goienetxea Uriarte A, Ruiz Zúñiga E, Urenda Moris M and Ng A H C (2017). How can decision makers be supported in the improvement of an emergency department? A simulation, optimization and data mining approach. Operations Research for Health Care 15:102-122. doi: 10.1016/j.orhc.2017.10.003.

Homer J B and Hirsch G B (2006). System dynamics modeling for public health: background and opportunities. American journal of public health 96(3):452-458. doi: 10.2105/AJPH.2005.062059.

Hulshof P J H, Kortbeek N, Boucherie R J, Hans E W and Bakker P J M (2012). Taxonomic classification of planning decisions in health care: a structured review of the state of the art in OR/MS. Health Systems 1:129-175.

Katsaliaki K and Mustafee N. (2011). Applications of simulation within the healthcare context." Journal of the Operational Research Society 62(8):1431-1451. doi: 10.1057/jors.2010.20.

Kivelä K, Elo S and Kääriäinen M (2018). Frequent attenders in primary health care: A concept analysis. International Journal of Nursing Studies 86:115-124. doi: https://doi.org/10.1016/j.ijnurstu.2018.06.003.

Kunc M, Mortenson M J and Vidgen R (2018). A computational literature review of the field of System Dynamics from 1974 to 2017. Journal of Simulation 12(2):115-127. doi: 10.1080/17477778.2018.1468950. 
LaCalle E and Rabin E (2010). Frequent Users of Emergency Departments: The Myths, the Data, and the Policy Implications. Annals of Emergency Medicine 56(1):42-48. doi: https://doi.org/10.1016/j.annemergmed.2010.01.032.

Lindgren B (2016). The Rise in Life Expectancy, Health Trends among the Elderly, and the Demand for Health and Social Care. Lund, Sweden: National Institute of Economic Research.

Linnéusson G, Ng A H C and Aslam T (2018). Towards strategic development of maintenance and its effects on production performance by using system dynamics in the automotive industry. International Journal of Production Economics. 200:151-169. doi: https://doi.org/10.1016/j.ijpe.2018.03.024.

Lyons G J and Duggan J (2015). System dynamics modelling to support policy analysis for sustainable health care. Journal of Simulation 9(2):129-139. doi: 10.1057/jos.2014.15.

Mielczarek B and Uziałko-Mydlikowska J (2012). Application of computer simulation modeling in the health care sector: A survey. SIMULATION 88(2): 197-216. doi: 10.1177/0037549710387802.

Moskop J C, Sklar D P, Geiderman J M, Schears R M and Bookman K J (2009). Emergency Department Crowding, Part 1 -Concept, Causes, and Moral Consequences. Annals of Emergency Medicine 53(5):605-611. doi: https://doi.org/10.1016/j.annemergmed.2008.09.019.

Repenning N P and Sterman J D (2001). Nobody Ever Gets Credit for Fixing Problems that Never Happened: Creating and Sustaining Process Improvement. California Management Review 43(4):64-88. doi: 10.1109/EMR.2002.1167285.

Salleh S, Thokala P, Brennan A, Hughes R and Booth A (2017). Simulation Modelling in Healthcare: An Umbrella Review of Systematic Literature Reviews. PharmacoEconomics 35(9):937-949. doi: 10.1007/s40273-017-0523-3.

Sargent R G (2011). Verification and validation of simulation models. In: Jain S, Creasey R, Himmelspach J, White K P and Fu M C (eds). Proceedings of the 2011 Winter Simulation Conference. Winter Simulation Conference: Phoenix, Arizona, US, pp 11-14.

Senge P M and Sterman J D (1992). Systems thinking and organizational learning: Acting locally and thinking globally in the organization of the future. European Journal of Operational Research 59(1):137-150. doi: 10.1016/0377-2217(92)90011-W.

Taylor K and Dangerfield B (2005). Modelling the feedback effects of reconfiguring health services. Journal of the Operational Research Society 56(6):659-675. doi: 10.1057/palgrave.jors.2601862.

United Nations (2017). World Population Prospects: The 2017 Revision, Key Findings and Advance Tables. United Nations.

\section{AUTHOR BIOGRAPHIES}

GARY LINNÉUSSON received a Ph.D. degree in Industrial Informatics (2018) from the University of Skövde in Sweden. His background is in Mechanical Engineering (2003) and has both an industrial and academic background from the fields of production engineering and maintenance using system dynamics and discrete event simulation. He is currently a researcher and lecturer at Jönköping University. His research interest is mainly on the problem structuring process using simulation and simulation-based optimization to its support in areas such as industry and healthcare. https://www.researchgate.net/profile/Gary_Linneusson

AINHOA GOIENETXEA URIARTE received a Ph.D. degree in Industrial Informatics (2019) from the University of Skövde in Sweden. Her background is in Computer Engineering (2004) and Industrial Management Engineering (2007), from the University of Mondragon in Spain. She has both an industrial and academic background in the areas of production engineering, innovation, and discrete event simulation. She is currently a researcher and lecturer at the University of Skövde. Her research interest is mainly on simulation and simulation-based optimization for decision support in healthcare and manufacturing system design and improvement. https:/www.his.se/en/about-us/Factsand-figures/staff/ainhoa_goienetxea/ 